\title{
Embolismo arterial agudo como forma de presentación clínica inicial de una hidatidosis diseminada: reporte de un caso
}

\author{
Acute arterial embolism as the clinical presentation of a disseminated \\ hydatidosis: case report
}

\author{
Dra. Estefanía Rossetti ${ }^{a}$ Dra. Anabella Boto ${ }^{b}$,Dr. Carlos González Cambaceres ${ }^{c}$, Dra. Silvina Ruvinsky ${ }^{d}$ y \\ Dr. Ezequiel Sagraye \\ Colaboradores: Dra. María Centeno y Dra. Jessica López Marti, Servicio de Anatomía Patológica, Hospital \\ "Prof. Dr. Juan P. Garrahan"
}

\section{RESUMEN}

La hidatidosis es una zoonosis producida por el parásito Echinococcus granulosus. En el ciclo zoonótico del parásito, el hombre es un huésped intermediario y sufre la enfermedad tras la ingesta de alimentos y agua contaminados por la materia fecal de animales infectados. En Argentina, la enfermedad constituye un problema importante de salud pública. Suele manifestarse con compromiso hepático y pulmonar. La afectación de otros órganos, que incluyen el corazón, es infrecuente.

Se presenta un caso de embolia arterial aguda como manifestación clínica inicial de una hidatidosis diseminada en un paciente pediátrico, a partir de la ruptura de un quiste hidatídico cardíaco.

Palabras clave: embolia y trombosis, equinococosis.

http:/ / dx.doi.org/10.5546/ aap.2018.e616

Texto completo en inglés:

http:/ / dx.doi.org/10.5546/ aap.2018.eng.e616

Cómo citar: Rossetti E, Boto A, González Cambaceres C, et al. Embolismo arterial agudo como forma de presentación clínica inicial de una hidatidosis diseminada: reporte de un caso. Arch Argent Pediatr 2018;116(4):e616-e620. a. Hospital "Prof. Dr. Juan P. Garrahan", Buenos Aires, Argentina.

b. Clínica Servicio de Emergencias.

c. Unidad de Cuidados Intensivos (UCI) 44.

d. Servicio de Epidemiología e Infectología.

Hospital "Prof. Dr. Juan P. Garrahan", Buenos Aires,

Argentina.

Correspondencia:

Dra. Estefanía Rossetti: estefania.rossetti86@gmail.com

Financiamiento: Ninguno.

Conflicto de intereses: Ninguno que declarar.

Recibido: 24-10-2017

Aceptado: 19-2-2018

\section{INTRODUCCIÓN}

La hidatidosis es una infección parasitaria producida por las larvas del Echinococcus granulosus, que se transmite por la ingestión de alimentos infectados y que se caracteriza por formar quistes en órganos, como el hígado, el pulmón, etc.

La infección por Echinococcus granulosus tiene una distribución geográfica universal con descripción de casos en todos los continentes. En América del Sur, existe en la mayoría de los países, pero, en Argentina, Bolivia, Brasil, Perú y Uruguay, es prevalente y constituye un importante problema para la salud pública. ${ }^{1}$

En Argentina, los casos de hidatidosis se distribuyen a lo largo de todo el territorio nacional, con mayor prevalencia en las zonas rurales, especialmente, en áreas de cría de ganado ovino y caprino. Se estima que el $30 \%$ del territorio nacional es asiento del ciclo zoonótico. Según el Boletín integrado de vigilancia 2017, en Argentina, durante los años 2016-2017, hubo 234 casos notificados y 116 confirmados. Las provincias con más casos notificados fueron Buenos Aires, Córdoba, Entre Ríos y Santa Fe. ${ }^{2}$

En los humanos, el compromiso es hepático en $67-89 \%$ de los casos y pulmonar en el $10-15 \%$ de los casos. La afectación de otros órganos, en conjunto, representa menos del $10 \%$ de los casos reportados. La afectación cardíaca es poco frecuente $(0,05-2 \%)$ y la ruptura de un quiste hidatídico cardíaco con posterior siembra en diferentes órganos ha sido raramente reportada. ${ }^{1}$

En el presente trabajo, se describe el caso de un paciente pediátrico con hidatidosis diseminada, que presentó un embolismo arterial agudo como manifestación clínica inicial de esta enfermedad.

\section{REPORTE DEL CASO}

Paciente de 15 años de edad, sexo masculino, oriundo de la localidad de Carmen de Areco, 
provincia de Buenos Aires, que habitaba en una zona rural. Junto con su familia, faenaban sus propios animales y alimentaban con los restos a los perros del campo. Ingresó por dolor epigástrico, seguido de parestesias en el miembro inferior derecho de menos de 24 horas de evolución. El dolor fue aumentando de intensidad con las horas, y se agregó un cambio de coloración de todo el miembro inferior afectado con la presencia de palidez marmórea. Asociado a esto, el paciente refería cefalea intensa. Consultaron en el hospital zonal, donde se sospechó trombosis arterial aguda del miembro inferior derecho. Se administró una dosis de heparina endovenosa, hidrocortisona endovenosa y se realizó la derivación a un centro de mayor complejidad.

Al ingresar al Servicio de Emergencias del Hospital "Prof. Dr. Juan P. Garrahan", el paciente se encontraba en regular estado general, somnoliento, hemodinámicamente estable, eupneico y normotenso. Al momento del examen físico, presentaba ruidos cardíacos normofonéticos, sin soplos. Auscultación respiratoria sin particularidades. Abdomen blando, depresible, sin visceromegalias. Miembro inferior derecho doloroso, frío, pálido, con ausencia de pulsos femoral, poplíteo, tibial posterior y pedio.

El diagnóstico inicial sugerido fue el de trombosis arterial aguda. Se solicitaron los siguientes exámenes complementarios:

FIgURA 1. Ecografía abdominal y renal bilateral

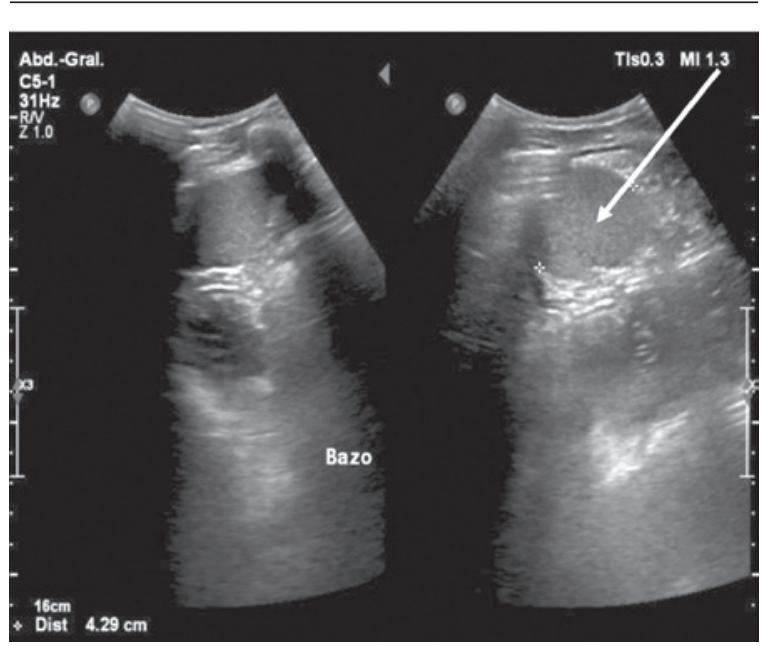

Imagen quística a nivel del polo superior del bazo (flecha).
- Ecografía doppler vascular; se observó una imagen ecogénica endoluminal en la arteria femoral común y femoral profunda derecha hasta el extremo proximal.

- Ecografía abdominal; se observó una imagen quística a nivel del polo superior del bazo, con diámetro de $11 * 10$ * $10 \mathrm{~cm}$. Hígado sin particularidades. Flujo aórtico conservado. (Figura $N^{\circ} 1$ ).

- Tomografía de cerebro sin contraste: sistema ventricular sin dilatación, sin desviación de la línea media y sin masa visible.

- Análisis de laboratorio, que informaba leucocitosis (glóbulos blancos -GB-: 14050/ $\mathrm{mm}^{3}$ ) de predominio neutrofílico (neutrófilos: 13340/ $\mathrm{mm}^{3}$ ), con linfopenia (linfocitos: 453 / $\mathrm{mm}^{3}$ ); el resto de las series eran normales; hepatograma, función renal y coagulograma dentro de los rangos normales. Serología para virus de la inmunodeficiencia humano (VIH) negativa.

- Ecocardiograma; se observó una imagen quística de $3 \mathrm{~cm}$ de diámetro dentro del ventrículo izquierdo (VI). Impresionaba haber comunicación entre el interior del quiste y la cavidad del VI. Función del VI conservada. Sin defectos estructurales congénitos. Sin derrame pericárdico $\left(\right.$ Figura $\left.N^{\circ} 2\right)$.

Considerando la epidemiología y las imágenes quísticas observadas, se sospechó como diagnóstico diferencial hidatidosis diseminada. Se

FIGURA 2. Ecocardiograma doppler

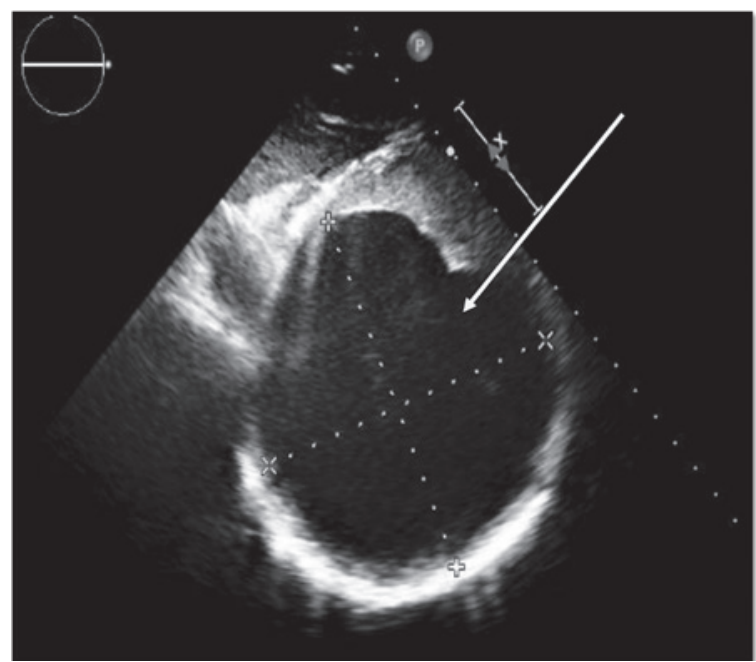

Imagen quística de $3 \mathrm{~cm}$ de diámetro dentro del ventrículo izquierdo (flecha). 
completaron los estudios con radiografía de tórax de frente, la cual no mostró imágenes patológicas.

Dentro de la clasificación ecográfica de la Organización Mundial de la Salud (OMS) para quistes hidatídicos, se catalogaron como tipo de quistes CL (por las siglas en inglés de cystic lesion): quiste de características inespecíficas, unilocular, de pared no visible y contenido líquido homogéneo anecogénico. Es indistinguible del quiste simple; considerado como un quiste hidatídico de muy temprano diagnóstico y fértil. ${ }^{1}$

Ingresaron muestras de ensayo por inmunoabsorción ligado a enzimas (enzymelinked immunosorbent assay; ELISA, por sus siglas en inglés) para hidatidosis en el Hospital, que fueron negativas. Al cabo de 24 horas del ingreso, se desarrolló una arteriotomía longitudinal de arteria femoral común y se realizó, según el parte quirúrgico, la extracción de material compatible con hidatidosis de $10 \mathrm{~cm}$ de longitud. (Figura $N^{\circ} 3$ ). Se comenzó, en dicha oportunidad, el tratamiento con albendazol para evitar mayor siembra hidatídica.

$\mathrm{Al}$ no presentar compromiso hemodinámico, el Servicio de Cirugía Cardiovascular decidió no realizar exéresis del quiste cardíaco al ingreso.

Se confirmó el diagnóstico de hidatidosis con la anatomía patológica de las muestras de la arteriotomía.
Debido a la gravedad del cuadro, el Servicio de Infectología decidió comenzar el tratamiento antibiótico combinado y agregó praziquantel (realizó la carga de cinco días y mantuvo luego el tratamiento semanal). A los 21 días, con buena evolución clínica, se decidió el ingreso al quirófano para la extracción de quiste cardíaco y esplénico.

Dicho procedimiento tardó seis horas y treinta minutos, y requirió una hora de circulación extracorpórea. Se realizó una ventriculotomía del VI y se resecó el quiste, el cual se encontraba adherido a la valva posterior de la válvula mitral. Presentó, durante la cirugía, fibrilación ventricular de cinco minutos de duración, que requirió desfibrilación y goteo de lidocaína. Se completó la cirugía con hemiesplenectomía. Ingresaron muestras para anatomía patológica y cultivo, y se corroboró la presencia de la membrana cuticular del quiste hidatídico. (Figura $N^{\circ} 4$ ).

Se repitió el ecocardiograma posquirúrgico, y se evidenció disfunción moderada del VI. Comenzó el tratamiento con carvedilol, espironolactona y enalapril. Se le aplicó un holter trece días tras la cirugía, que informó ritmo sinusal permanente que alternaba con ritmo auricular en horas del sueño, con rango cronotrópico en el límite superior. Extrasístoles supraventriculares aisladas, extrasístoles ventriculares aisladas. No se reportaron síntomas durante el estudio. El Servicio de Cardiología decidió no realizar el tratamiento antiarrítmico.

FIGURE 4. Resección de quiste hidatídico esplénico

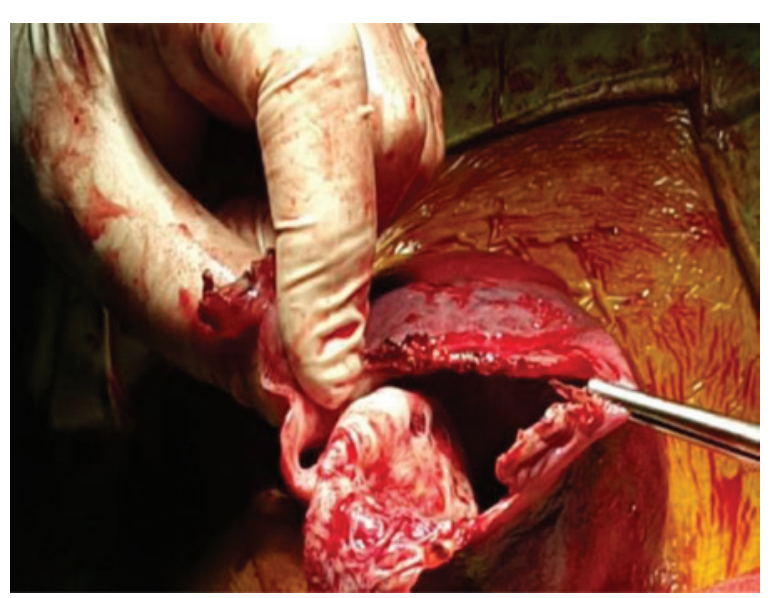

FIGURE 3. Muestra de material extraído del interior de la arteria femoral derecha en la primera cirugía (arteriotomía femoral)

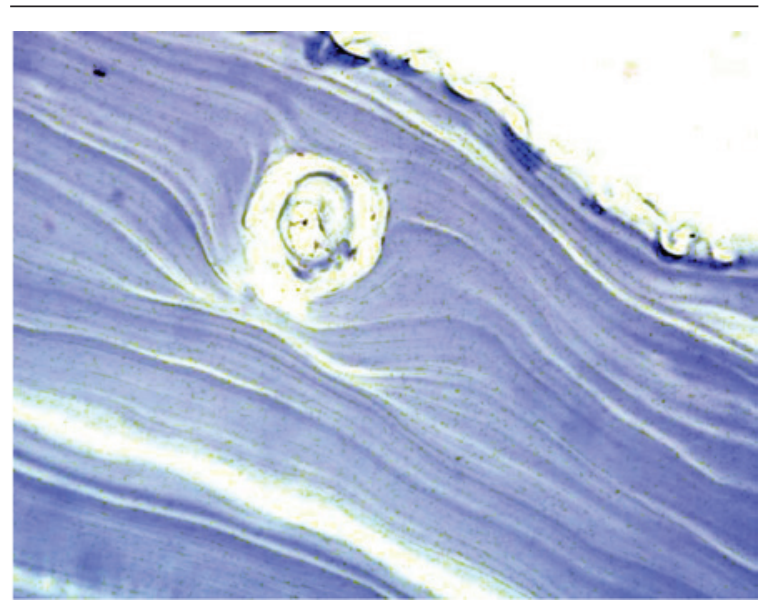

En la sección histológica, se observa una pared

correspondiente al quiste hidatídico, constituido por una membrana laminar que, en la superficie, exhibe parcialmente la capa germinativa y, en su interior, se reconoce un escólice. (H\&E 10X). 
Por buena evolución clínica, se decidió otorgar el egreso hospitalario tras haber permanecido internado por treinta y ocho días.

Continúa el seguimiento por el Servicio de Clínica Pediátrica, Infectología y Cardiología. Mantiene el tratamiento cardiológico con carvedilol, espironolactona y enalapril. Cumplió el tratamiento antibiótico con praziquantel semanal por un período de quince meses y albendazol diario por un período de dieciocho meses. Tras dos años de seguimiento hospitalario, por el momento, no presentó recidivas de la enfermedad, con buena respuesta al tratamiento instaurado. La última ecografía abdominal mostró bazo residual y no se evidenciaron imágenes quísticas.

\section{DISCUSIÓN}

La hidatidosis cardíaca es una presentación infrecuente de la enfermedad, pero con alta morbimortalidad asociada.

E. granulosus accede al corazón a través de la circulación coronaria habitualmente, pero también un foramen oval permeable, la circulación pulmonar, los linfáticos intestinales, el conducto torácico, las venas cavas y las venas hemorroidales pueden ser otros lugares de acceso. $^{3}$

La localización más frecuente de afectación cardíaca es el VI (55-60\%); la afectación del ventrículo derecho y el septum interventricular es menos habitual (10-15\% y 4\%, respectivamente). ${ }^{3}$

Generalmente, se presenta como único quiste intramiocárdico izquierdo $\mathrm{y}$, en la mayoría de los casos, hay afectación extracardíaca. En el trabajo de Miralles et ál., existe hasta un 55\% de afectación extracardíaca asociada, y la más frecuente es la hepática. La mayoría de los quistes son asintomáticos, pero de alto riesgo de ruptura espontánea y, por ello, de complicaciones, desde embólicas hasta cuadros de anafilaxia grave. ${ }^{4}$

Los quistes del corazón izquierdo tienden a crecer hacia el epicardio y romperse en el espacio epicárdico. Los quistes en el corazón derecho tienden a expandirse en forma intracavitaria y subendocárdica, y los del ventrículo derecho tienden más frecuentemente a romperse y, por ende, a desarrollar embolias pulmonares, anafilaxia o muerte súbita. ${ }^{5}$

Cuando está afectado el septum interventricular, lo característico son las arritmias. Los bloqueos en la conducción son posibles, como así también la obstrucción del tracto de salida de los ventrículos derecho o izquierdo. ${ }^{6}$
Aunque se han descrito casos de compromiso arterial por fístulas cistovasculares, el compromiso arterial se desarrolla, generalmente, a posteriori de la ruptura de un quiste cardíaco y su embolización secundaria. Sin embargo, la embolización arterial secundaria a un quiste hidatídico cardíaco, como en este caso, es una extrema rareza. En la bibliografía, han sido reportados pocos casos en los que se describe la presentación de hidatidosis en forma de embolia arterial periférica secundaria a quistes intracardíacos. ${ }^{7-14}$ De estos, la mayoría se presentan en adultos.

\section{CONCLUSIÓN}

A pesar de ser la embolia arterial aguda una forma de presentación atípica infrecuente de la hidatidosis diseminada, en nuestro país, zona endémica de hidatidosis, deberá considerarse como diagnóstico diferencial ante tromboembolismo arterial o masa cardíaca en los pacientes con imágenes y epidemiología compatible.

\section{Agradecimientos}

Servicio de Cirugía Cardiovascular, Servicio de Cirugía General y Servicio de Diagnóstico por Imágenes del Hospital "Prof. Dr. Juan P. Garrahan".

Dr. Tomás Orduna, médico infectológo. Jefe del Servicio de Patologías Regionales y Medicina Tropical. Hospital de Infecciosas Francisco J. Muñiz.

\section{REFERENCIAS}

1. Enfermedades infecciosas. Hidatidosis. Guía para el Equipo de Salud. Buenos Aires: Ministerio de Salud de la Nación; 2012. [Acceso: 21 de febrero de 2018]. Disponible en: www. msal.gob.ar/images/stories/epidemiologia/pdf/guiamedica-hidatidosis.pdf.

2. Ministerio de Salud de la Nación. Boletín integrado de Vigilancia. 2017;(356)SE 16. [Acceso: 21 de febrero de 2018]. Disponible en: http: / / www.msal.gob.ar/images/stories / boletines/boletin_integrado_vigilancia_N356-SE16.pdf.

3. Ipek G, Omeroglu S. Goksedef D, et al. Large cardiac hydatid cyst in the interventricular septum. Tex Heart Inst J 2011; 38(6):719-22.

4. Miralles A, Bracamonte L, et al. Cardiac echinococcosis. Surgical treatment and results. J Thorac Cardiovasc Surg 1994; 107(1):184-90.

5. Dursun M, TerzibasiogluE, Yilmaz R, etal. Cardiachydatid disease: CT and MRI findings. AJR Am J Roentgenol 2008; 190(1):226-32.

6. Muthu SK, Thiagarajan A, Govindarajan S, et al. Hydatid cyst of the cardiac interventricular septum: report of two cases. Br J Radiol 2007; 80(959):e278-82.

7. Ceyran H, Tasdemir K, Tezcaner T, et al. A rare cause of peripheral arterial embolism: ruptured cardiac hydatid cyst. Vasa 2002; 31(2):129-31.

8. Kardaras F, Kardara D, Tselikost A, et al. Fifteen year surveillance of echinococcal heart disease from a referral hospital in Greece. Eur Heart J 1996;17(8):1265-70. 
9. Papacharalambous G, Panoussis P, Andrikopoulos V, et al. Chronic Arterial Occlusion Produced by Hydatid Cyst Development in the Lumen of the Femoral Artery. Eur J Vasc Endovasc Surg 1998; 15(6):544-6.

10. Rosenberg T, Panayiotopoulos YP, Bastounis E, et al. Acute abdominal aorta embolism caused by primary cardiac echinococcus cyst. Eur J Vasc Surg 1993; 7(5):582-5.

11. Shields DA. Multiple emboli in hydatid disease. BMJ 1990; 301(6745):213-4.

12. Zandi B, Fard MM, Farzad Nia M, et al. Interventricular septum hydatid cyst causing ileofemoral artery embolism and ischemic syndrome of the lower limb. ARYA Atheroscler 2007; 3(1):38-41.
13. Tacyildiz IH. Acute Abdominal Aortic Embolism Caused by Primary Cardiac Echinococcus Cyst: A Case Report. Vasc Endovascular Surg 1999; 33(1):119-22.

14. Öztürk M, Sığırcı A, Dağlı AF. A rare cause of embolism in the popliteal artery of an adolescent: ruptured cardiac hydatid cyst. Anatol J Cardiol 2015; 15(6):E20.

15. Melonari P, Bellone L. Hidatidosis. En: Comité Nacional de Infectología. Consenso Sobre Enfermedades Infecciosas Regionales en la Argentina. Recomendaciones de la Sociedad Argentina de Pediatría. 2012-2013:8-38. [Acceso: 21 de febrero de 2018]. Disponibleen:http:/ / www.sap.org. ar/docs / profesionales / consensos / consenso_patologia_ regional.pdf. 